\title{
From dislocation motion to an additive velocity gradient decomposition, and some simple models of dislocation dynamics*
}

\author{
Amit Acharya \\ Carnegie Mellon University, \\ Pittsburgh, PA 15213, USA \\ E-mail: acharyaamit@cmu.edu \\ Xiaohan Zhang \\ Carnegie Mellon University, \\ Pittsburgh, PA 15213, USA \\ E-mail: zxiaohan@andrew.cmu.edu
}

June 4, 2014

\begin{abstract}
A mathematical theory of time-dependent dislocation mechanics of unrestricted geometric and material nonlinearity is reviewed. Within a 'small deformation' setting, a suite of simplified, but interesting, models, namely a nonlocal Ginzburg Landau, a nonlocal level set, and a nonlocal generalized Burgers equation are derived. In the finite deformation setting, it is shown that an additive decomposition of the total velocity gradient into elastic and plastic parts emerges naturally from a micromechanical starting point that involves no notion of plastic deformation but only the elastic distortion, material velocity, dislocation density and the dislocation velocity. Moreover, a plastic spin tensor emerges naturally as well.
\end{abstract}

Dedicated to Professor Luc Tartar on his $65^{\text {th }}$ birthday.

\section{Introduction}

We describe a partial differential equation (pde) based model of the timedependent mechanics of dislocations which are defects of compatibility of elastic

* In Proceedings of the International Conference on Nonlinear and Multiscale Partial Diffferential Equations: Theory, Numerics and Applications held at Fudan University, Shanghai, September 16-20, 2013, in honor of Luc Tartar. Editors Ph. G. Ciarlet and Ta-Tsien Li, Series in Contemporary Applied Mathematics. Higher Education Press (Beijing), and World Scientific (Singapore), 2014. 
deformation. The classical treatment due to Volterra describes such defects as non-square-integrable singularities in a static setting. Due to these infiniteenergy singularities, what PDE dynamics should represent such objects is not clear; there is a version called Discrete Dislocation Dynamics (Lepinoux and Kubin [1], Amodeo and Ghoniem [2], Van der Giessen and Needleman [3], Zbib et al. [4]) that treats these linear elastic singularities as discrete objects with their short-range interactions (e.g. collisions, annihilations) treated through empirical rules. The theory of Continuously Distributed Dislocations due to Kröner, [6] (and earlier references therein), Mura [7], Fox [8], and Willis [9] provides the beginnings of a dynamical pde model. This model has been completed, generalized, and understood as a rigorous, continuum thermomechanical model of dislocation dynamics and its collective behavior in [10, 11, 12, 13, 14, 15]. In this paper, we derive simplified, but exact, models of the full three-dimensional theory that have been shown, through numerical computation to be reported elsewhere, to sustain localized but non-singular stress concentrations in unloaded bodies bearing much similarity to the Volterra dislocation stress fields, and represent their motion and interaction. We also report an interesting implication of the finite deformation theory that sheds fundamental light on an often controversial aspect of the phenomenological theory of finite strain plasticity, namely, the issue of the physically appropriate mathematical representation of the decomposition of the total deformation(rate) into elastic and plastic parts.

This paper is organized as follows: in Section 2 we briefly recall the basic equations of FDM in the 'small deformation' setting. In Section 3 we derive a family of simplified models suitable for mathematical analysis. In Section 4, we recall the equations of FDM without any restrictions of material or geometric nonlinearity and show that this fundamental viewpoint unambiguously points to a specific decomposition of the total velocity gradient into elastic and plastic parts as well as the identification of plastic spin, the latter even in situations that invoke no crystalline microstructure.

\section{FDM 'small deformation' theory}

We use the following notation: In rectangular Cartesian coordinates and components,

$$
\begin{aligned}
& (\boldsymbol{A} \times \boldsymbol{v})_{i m}=e_{m j k} A_{i j} v_{k} ;(\operatorname{div} \boldsymbol{A})_{i}=A_{i j, j} ;(\operatorname{curl} \boldsymbol{A})_{i m}=e_{m j k} A_{i k, j} ; \\
& (\boldsymbol{a} \cdot \boldsymbol{b})=a_{i} b_{i} ;(\boldsymbol{A}: \boldsymbol{B})=A_{i j} B_{i j} ;(\boldsymbol{a} \otimes \boldsymbol{b})_{i j}=a_{i} b_{j}
\end{aligned}
$$

where $e_{m j k}$ is a component of the third-order alternating tensor $\boldsymbol{X}$. The symbol div represents the divergence, grad the gradient, and divgrad the Laplacian. A superposed 'dot' represents a material time-derivative.

In the following, we will have occasion to refer to a quantity called a Burgers vector. For the present purposes where we have in mind a common mathematical structure for dislocation mechanics in crystalline and amorphous materials as well as geophysical rupture dynamics, it is best understood as the vector residue 
obtained on integrating the elastic distortion field along a closed curve - note that when the distortion is a gradient (as in elasticity without dislocations) the residue vanishes. A closed contour encircling a fault front terminating a slipped region in a fault is one example of such a contour with non-vanishing residue. By Stokes' theorem, the curl of the elastic distortion field then serves as an areal density of lines carrying a vectorial attribute (the Burgers vector). It is this field that we refer to as the dislocation density (tensor) field. The complete set of equations is

$$
\left.\begin{array}{l}
\operatorname{curl} \boldsymbol{\chi}=\boldsymbol{\alpha}=\operatorname{curl} \boldsymbol{U}^{e}=-\operatorname{curl} \boldsymbol{U}^{p} \text { (elastic incompatibility) } \\
\operatorname{div} \boldsymbol{\chi}=\mathbf{0} \\
\operatorname{div}(\operatorname{grad} \dot{\boldsymbol{z}})=\operatorname{div}(\boldsymbol{\alpha} \times \boldsymbol{V}) \\
\boldsymbol{U}^{e}:=\operatorname{grad}(\boldsymbol{u}-\boldsymbol{z})+\boldsymbol{\chi} ; \boldsymbol{U}^{p}:=\operatorname{grad} \boldsymbol{z}-\boldsymbol{\chi} \\
\operatorname{div}\left[\boldsymbol{T}\left(\boldsymbol{U}^{e}\right)\right]+\boldsymbol{b}=\rho \ddot{\boldsymbol{u}} \text { (balance of linear momentum) } \\
\dot{\boldsymbol{\alpha}}=-\operatorname{curl}(\boldsymbol{\alpha} \times \boldsymbol{V}) \text { (conservation of Burgers vector content) }
\end{array}\right\} \text { on } R
$$

Here, $R \subset \mathbb{R}^{3}$ is a region of space that represents the body and the various fields are defined as follows. $\chi$ is the incompatible part of the elastic distortion tensor $\boldsymbol{U}^{e}, \boldsymbol{u}$ is the total displacement field, and $\boldsymbol{u}-\boldsymbol{z}$ is a vector field whose gradient is the compatible part of the elastic distortion tensor. $\boldsymbol{U}^{p}$ is the plastic distortion tensor. $\boldsymbol{\alpha}$ is the dislocation density tensor, and $\boldsymbol{V}$ is the dislocation velocity vector. $\boldsymbol{\alpha} \times \boldsymbol{V}$ (plastic strain rate with physical dimensions of time ${ }^{-1}$ ) represents the flow of Burgers vector carried by the dislocation density field moving with velocity $\boldsymbol{V}$ relative to the material. For the sake of intuition, indeed, when $\boldsymbol{\alpha}=\boldsymbol{b} \otimes \boldsymbol{l}$ with $\boldsymbol{b}$ perpendicular to $\boldsymbol{l}$ (an edge dislocation) and $\boldsymbol{V}$ in the plane spanned by $\boldsymbol{b}$ and $\boldsymbol{l}, \boldsymbol{\alpha} \otimes \boldsymbol{V}$ represents a simple shearing (strain rate) in the direction of $\boldsymbol{b}$ on planes normal to $\boldsymbol{l} \times \boldsymbol{V}$. The argument of the div operator in Eqs. (2.15) is the (symmetric) stress tensor, $\boldsymbol{b}$ is the body force density, and the functions $\boldsymbol{V}, \boldsymbol{T}$ are constitutively specified. All the statements in the governing equations are fundamental statements of kinematics or conservation. In particular, Eqs. $\left(2.1_{6}\right)$ is a purely geometric statement of conservation of Burgers vector content carried by a density of lines (see [11] for a derivation) and Eqs. (2.1 $)_{5}$ is the balance of linear momentum.

As for boundary conditions,

$$
\left.\begin{array}{l}
\chi \boldsymbol{n}=0 \\
(\operatorname{grad} \dot{\boldsymbol{z}}-\boldsymbol{\alpha} \times \boldsymbol{V}) \boldsymbol{n}=0
\end{array}\right\} \text { on } \partial R
$$

are imposed along with standard conditions on displacement and/or traction.

A natural boundary condition associated with Eqs. $(2.1)_{6}$, important in the context of modeling rupture dynamics in faults as an interface condition with purely elastic material, is

$$
(\boldsymbol{\alpha} \times \boldsymbol{V}) \times \boldsymbol{n}=0 .
$$

Often, one is interested in coarse length and time scale behavior of the system (2.1). For such a purpose, an elementary filtering approach can be adopted to 
convert FDM to Mesoscale FDM (MFDM). For a microscopic field $\boldsymbol{f}$ given as a function of space and time, making use of a standard filtering technique e.g. Babic [16], one defines the mesoscopic space-time average field $\bar{f}$ as

$$
\bar{f}:=\frac{1}{\int_{I(t)} \int_{\Gamma(x)} w\left(x-x^{\prime}, t-t^{\prime}\right) d x^{\prime} d t^{\prime}} \int_{\Lambda} \int_{B} w\left(x-x^{\prime}, t-t^{\prime}\right) f\left(x^{\prime}, t^{\prime}\right) d x^{\prime} d t^{\prime}
$$

where $B$ is the body and $\Lambda$ a sufficiently large interval of time. $\Gamma(x)$ is a bounded region within the body around the point $x$ with linear dimension of the order of the spatial resolution of the macroscopic model we seek, $I(t)$ is a bounded interval in $\Lambda$ containing $t$. The averaged field $\bar{f}$ obtained in this manner is a weighted, space-time, running average of the microscopic field $f$ over regions whose scale is determined by the scale of spatial and temporal resolution of the averaged model. The weighting function $w$ is non-dimensional, smooth in the variables $x, x^{\prime}, t, t^{\prime}$ and, for fixed $x$ and $t$, has support only in $\Gamma(x) \times I(t)$ when viewed as a function of $\left(x^{\prime}, t^{\prime}\right)$. Applying this operator to the system (2.1), we obtain an exact set of equations for the averages given as

$$
\begin{aligned}
& \operatorname{curl} \overline{\boldsymbol{\chi}}=\overline{\boldsymbol{\alpha}} \\
& \operatorname{div} \overline{\boldsymbol{\chi}}=\mathbf{0} \\
& \operatorname{divgrad} \dot{\overline{\boldsymbol{z}}}=\operatorname{div}\left(\overline{\boldsymbol{\alpha}} \times \overline{\boldsymbol{V}}+\boldsymbol{L}^{p}\right) \\
& \overline{\boldsymbol{U}}^{e}:=\operatorname{grad}(\overline{\boldsymbol{u}}-\overline{\boldsymbol{z}})+\overline{\boldsymbol{\chi}} \\
& \rho \ddot{\overline{\boldsymbol{u}}}=\operatorname{div} \overline{\boldsymbol{T}} \\
& \dot{\overline{\boldsymbol{\alpha}}}=-\operatorname{curl}\left(\overline{\boldsymbol{\alpha}} \times \overline{\boldsymbol{V}}+\boldsymbol{L}^{p}\right)
\end{aligned}
$$

where $\boldsymbol{L}^{p}$ is defined as

$$
\boldsymbol{L}^{p}(\boldsymbol{x}, t):=\overline{(\boldsymbol{\alpha}-\overline{\boldsymbol{\alpha}}(\boldsymbol{x}, t)) \times \boldsymbol{V}}=\overline{\boldsymbol{\alpha} \times V}(\boldsymbol{x}, t)-\overline{\boldsymbol{\alpha}}(\boldsymbol{x}, t) \times \overline{\boldsymbol{V}}(\boldsymbol{x}, t) .
$$

$\overline{\boldsymbol{\alpha}}$ is the mathematical embodiment of what is physically referred to alternatively as the geometrically-necessary-dislocation density (GND), polar dislocation density, or excess dislocation density. We define the nonlocal object

$$
\boldsymbol{\beta}\left(\boldsymbol{x}, \boldsymbol{x}^{\prime}, t, t^{\prime}\right)=\boldsymbol{\alpha}\left(\boldsymbol{x}^{\prime}, t^{\prime}\right)-\boldsymbol{\alpha}(\boldsymbol{x}, t)
$$

as the statistical dislocation density (SD) around $\boldsymbol{x} . \overline{\boldsymbol{\alpha} \times \boldsymbol{V}}$ is the mathematical representation of the plastic strain rate (rate of permanent deformation) that is produced at a macroscopic point due to the motion of dislocations. Clearly, the exact formula (2.4) above suggests that it can be interpreted as a sum of a plastic strain rate produced by the GND distribution imagined to be moving with a velocity $\overline{\boldsymbol{V}}$ and the averaged plastic strain rate produced by the SD distribution, $\overline{\boldsymbol{L}}^{p}$. In particular, the latter can be non-vanishing even when $\overline{\boldsymbol{\alpha}}=\mathbf{0}$, a fact of utmost importance in a model of averaged behavior. An elementary, idealized, realization of such a situation corresponds to a uniformly expanding square loop within $\Gamma(x)$. Here, $\boldsymbol{b}$ is assumed to be the Burgers vector density per unit area, uniform along the loop, and $\boldsymbol{l}$ the unit line direction at each point of the loop; 
opposite edges of the loop cancel each other in the spatial averaging yielding $\overline{\boldsymbol{\alpha}}=\mathbf{0}$. The velocity is assumed to lie in a slip plane and point outwards with respect to the loop with uniform magnitude; along individual parallel sides of the loop with opposite direction, $\boldsymbol{\alpha} \times \boldsymbol{V}=\boldsymbol{b} \otimes(\boldsymbol{l} \times \boldsymbol{V})$ is identical (since $\boldsymbol{l}$ and $\boldsymbol{V}$ both change sign going from one side to the other). Therefore, $\overline{\boldsymbol{\alpha}} \times \overline{\boldsymbol{V}}=\mathbf{0}$ but $\boldsymbol{L}^{p}=\overline{\boldsymbol{\alpha} \times \boldsymbol{V}}-\overline{\boldsymbol{\alpha}} \times \overline{\boldsymbol{V}} \neq \mathbf{0}$.

In fact, for 'large' enough spatial scales of averaging $(\sim 1 \mathrm{~mm})$, dislocations, by virtue of being stress-inducing entities, tend to produce distributions with $\overline{\boldsymbol{\alpha}} \approx \mathbf{0}$. However, from the above example it is clear that the averaged plastic strain rate of such distributions, when moving, is non-zero. When the averaging scale is 'small' $(\sim 1 \mu \mathrm{m})$, GNDs begin to emerge and produce significantly different behavior than at macroscopic scales such as length scale effects in strength and intermittent plastic flow.

\section{A 2-D Straight edge dislocation model from FDM}

We consider the geometry shown in Fig. 1;

$$
\begin{aligned}
\Omega & =\{(x, y):(x, y) \in[-W,+W] \times[-H,+H]\}, \\
L & =\{(x, y):(x, y) \in[-W,+W] \times[-b,+b]\}, \\
0 & <b<H, \quad W>0 .
\end{aligned}
$$

In our notation we use $x \equiv x_{1}$ and $y \equiv x_{2}$, synonymously. The model may be viewed as a composite comprising two outer regions, $\Omega \backslash L$, whose stress response is purely linear elastic, and the layer $L$, of width $2 b$, whose response is elasticplastic and where edge dislocations exist and FDM is active. The displacement field $\boldsymbol{u}$ is continuous on the entire domain. We interpret the slip field in the layer as:

$$
s(x, t)=\int_{-b}^{+b} u_{1,2}(x, y, t) d y=u_{1}(x, b, t)-u_{1}(x,-b, t) .
$$

This field does not play an explicit role in the constitutive modeling, due to the latter's inherently bulk nature. The dissipation on the whole body, defined as the difference of the rate of working of external forces and the rate of stored energy in the body, arises only from the layer (since everywhere else the body is elastic). Assume a stored energy density function of the form

$$
\psi\left(\boldsymbol{\epsilon}^{e}, \boldsymbol{\alpha}\right)+\eta\left(\boldsymbol{U}^{p}\right)
$$

with stress given by $\boldsymbol{T}=\partial \psi / \partial \boldsymbol{\epsilon}^{e}$, where $\boldsymbol{\epsilon}^{e}$ is the symmetric part of the elastic distortion $\boldsymbol{U}^{e}$. The function $\psi$ is assumed to be positive-definite quadratic in $\boldsymbol{\epsilon}^{e}$ and the function $\eta$ is multi-well non-convex, meant to embody the fact that certain plastically strained states are energetically more favorable than others, 
as well as endow the energy function with barriers to slip. Together, these two functions enable the robust modeling of overall total strain distributions in the layer displaying localized, smooth transitions between slipped and unslipped regions (or between the preferred strain states encoded in $\eta$ ). This crucially requires adding an energetic penalty to the development of high values of the dislocation density $\boldsymbol{\alpha}$, referred to as a core energy. In effect, the linear elastic stress and the core term tend to prevent a sharp discontinuity and the driving force from the non-convex $\eta$ term promotes the discontinuity, and it is the balance between these thermodynamic forces that sets the dislocation core width at equilibrium. Interestingly, it can be shown that while in the presence of just one component of plastic distortion only the linear elastic term suffices to give a finite core width (paralleling a fundamental result due to Peierls [17]), with more than one component, the core regularization from the $\boldsymbol{\alpha}$ term is essential $[18,19]$. It is to be noted that the core energy is a fundamental physical ingredient of our model and not simply a mathematical regularization. In general, it is not expected to have the simple 'isotropic' form assumed here and, in fact, its characterization furnishes our model with a direct route of making contact with sub-atomic, quantum mechanical physics.

The dissipation in the model can be written as

$$
\begin{aligned}
\mathrm{D}= & \int_{L}\left(\boldsymbol{T}-\frac{\partial \eta}{\partial \boldsymbol{U}^{p}}\right): \dot{\boldsymbol{U}}^{p} d v+\int_{L} \frac{\partial \psi}{\partial \boldsymbol{\alpha}}: \operatorname{curl}(\boldsymbol{\alpha} \times \boldsymbol{V}) d v \\
= & \int_{L}\left(\boldsymbol{T}-\frac{\partial \eta}{\partial \boldsymbol{U}^{p}}\right):(\boldsymbol{\alpha} \times \boldsymbol{V}) d v+\int_{L} \operatorname{curl}\left(\frac{\partial \psi}{\partial \boldsymbol{\alpha}}\right): \boldsymbol{\alpha} \times \boldsymbol{V} d v \\
& +\int_{\partial L} \frac{\partial \psi}{\partial \boldsymbol{\alpha}}:(\boldsymbol{\alpha} \times \boldsymbol{V}) \times \boldsymbol{n} d a
\end{aligned}
$$

where $\boldsymbol{n}$ is the outward unit normal field to the body.

In the layer assume the ansatz

$$
\begin{aligned}
\boldsymbol{U}^{p}(x, y, t) & =U_{12}^{p}(x, y, t) e_{1} \otimes e_{2}+U_{22}^{p}(x, y, t) e_{2} \otimes e_{2} \\
& :=\phi(x, t) e_{1} \otimes e_{2}+\omega(x, t) e_{2} \otimes e_{2}
\end{aligned}
$$

where the functions $\phi(x, t), \omega(x, t)$ need to be defined.

Then

$$
\boldsymbol{\alpha}(x, y, t)=-\operatorname{curl} \boldsymbol{U}^{p}(x, y, t)=-\phi_{x}(x, t) e_{1} \otimes e_{3}-\omega_{x}(x, t) e_{2} \otimes e_{3}
$$

and

$$
\operatorname{curl} \boldsymbol{\alpha}(x, y, t)=\phi_{x x}(x, t) e_{1} \otimes e_{2}+\omega_{x x}(x, t) e_{2} \otimes e_{2}
$$

where a subscript $x$ or $t$ represents partial differentiation with respect to $x$ or $t$, respectively. In keeping with the 2-d nature of this analysis and the constraint posed by the layer on the dislocation velocity, we assume

$$
\boldsymbol{V}(x, y, t)=V_{1}(x, y, t) \boldsymbol{e}_{1}:=v(x, t) \boldsymbol{e}_{1}
$$

where $v(x, t)$ needs to be defined. 
Note that with these assumptions, the boundary term in the dissipation vanishes for the horizontal portions of the layer boundary. We also assume

$$
\frac{\partial \psi}{\partial \boldsymbol{\alpha}}=\epsilon \boldsymbol{\alpha}
$$

where $\epsilon$ is a parameter with physical dimensions of stress $\times$ length $^{2}$ that introduces a length scale and essentially sets the width of the dislocation core, at equilibrium. For specific simplicity in this problem, we impose $\boldsymbol{\alpha}=\mathbf{0}$ on vertical portions of the layer boundary by imposing $\phi_{x}( \pm W, t)=\omega_{x}( \pm W, t)=0$.

With the above ansatz, the conservation law $\dot{\boldsymbol{\alpha}}=-\operatorname{curl}(\boldsymbol{\alpha} \times \boldsymbol{V})$ reduces to

$$
\begin{array}{llll}
\phi_{t}(x, t)=-\phi_{x} v(x, t) & \text { or } & \hat{\alpha}_{1 t}=-\left(\hat{\alpha}_{1} v\right)_{x} \\
\omega_{t}(x, t)=-\omega_{x} v(x, t) & \text { or } & \hat{\alpha}_{2 t}=-\left(\hat{\alpha}_{2} v\right)_{x}
\end{array}
$$

where $\hat{\alpha}_{1}:=-\phi_{x}=\alpha_{13}$ and $\hat{\alpha}_{2}:=-\omega_{x}=\alpha_{23}$. which define the evolution equations for plastic distortion components $\phi, \omega$ once $v$ is defined as a function of $(x, t)$.

We now consider the dissipation

$$
\begin{aligned}
\mathrm{D} & =\int_{L} V_{1}\left\{e_{1 j 3}\left(T-A+\epsilon(\operatorname{curl} \boldsymbol{\alpha})^{T}\right)_{j r} \alpha_{r 3}\right\} d v \quad A_{j r}:=\left(\frac{\partial \eta}{\partial \boldsymbol{U}^{p}}\right)_{j r} \\
& =\int_{L} v(x, t)\left\{\begin{array}{c}
{\left[T_{12}(x, y, t)-A_{12}(x, t)+\epsilon \phi_{x x}(x, t)\right]\left(-\phi_{x}(x, t)\right)} \\
+\left[T_{22}(x, y, t)-A_{22}(x, t)+\epsilon \omega_{x x}(x, t)\right]\left(-\omega_{x}(x, t)\right)
\end{array}\right\} d v .
\end{aligned}
$$

We make the choice

$$
\begin{aligned}
v(x, t):= & \frac{-1}{B l^{m-1}|\hat{\boldsymbol{\alpha}}|^{m}(x, t)}\left\{\begin{array}{r}
\phi_{x}(x, t)\left[\tau(x, t)-\tau^{b}(x, t)+\epsilon \phi_{x x}(x, t)\right] \\
+\omega_{x}(x, t)\left[\sigma(x, t)-\sigma^{b}(x, t)+\epsilon \omega_{x x}(x, t)\right]
\end{array}\right\} \\
& m=0,1 \text { or } 2 \\
\tau(x, t):= & \frac{1}{2 b} \int_{-b}^{b} T_{12}(x, y, t) d y ; \quad \tau^{b}:=A_{12} \\
\sigma(x, t):= & \frac{1}{2 b} \int_{-b}^{b} T_{22}(x, y, t) d y ; \quad \sigma^{b}:=A_{22}
\end{aligned}
$$

(i.e. kinetics in the direction of driving force (Rice, $1971[20]$ ), in the context of crystal plasticity theory), where $\hat{B}=B l^{m-1}|\hat{\alpha}|^{m}$ is a non-negative drag coefficient that characterizes the energy dissipation by specifying how the dislocation velocity responds to the applied driving force locally and $l$ is an internal length scale, e.g. Burgers vector magnitude of crystals. For simplicity, we have taken the drag to be a scalar but in general its inverse, the mobility, could be a positive-semidefinite tensor. In general, it is in $\hat{B}$ that one would like to model the effect of layer structural inhomogeneities impeding dislocations as well as the effect of other microscopic mechanisms of energy dissipation during dislocation motion. For $m=1, B$ has physical dimensions of stress $\times$ time $\times$ length $^{-1}$, and introduces another length scale related to kinetic effects. 
Then the dissipation becomes

$$
\begin{aligned}
\mathrm{D}= & \int_{L} \frac{1}{B l^{m-1}|\alpha|^{m}(x, t)}\left\{\begin{array}{r}
\phi_{x}(x, t)\left[\tau(x, t)-\tau^{b}(x, t)+\epsilon \phi_{x x}(x, t)\right] \\
+\omega_{x}(x, t)\left[\sigma(x, t)-\sigma^{b}(x, t)+\epsilon \omega_{x x}(x, t)\right]
\end{array}\right\} d x d y \\
& +\mathrm{R},
\end{aligned}
$$

where

$$
\mathrm{R}=\int_{x=-W}^{x=+W}-v(x, t)\left\{\begin{array}{c}
\phi_{x}(x, t) \int_{-b}^{b}\left[T_{12}(x, y, t)-\tau(x, t)\right] d y \\
+\omega_{x}(x, t) \int_{-b}^{b}\left[T_{22}(x, y, t)-\sigma(x, t)\right] d y
\end{array}\right\} d x
$$

Recalling the definitions of the layer-averaged stresses $\tau, \sigma$ in (3.1), we observe that

$$
\mathrm{R}=0 \text { and } \mathrm{D} \geq 0 .
$$

To summarize, within the class of kinetic relations for dislocation velocity in terms of driving force, positive dissipation along with the (global) conservation of Burgers vector content governs the nonlinear and nonlocal slip dynamics of the model. Essentially, slip gradients induce stress and elastic energy and the evolution of the dislocation is a means for the media to relieve this energy, subject to conservation of mass, momentum, energy, and Burgers vector.

To further simplify matters, we make the assumption that $\omega \equiv 0$, i.e. no normal plastic strain in the composite layer. Suppressing the argument $(x, t)$, the governing equation for the plastic shear strain now becomes

$$
\phi_{t}=\frac{\left|\phi_{x}\right|^{2}}{B l^{m-1}|\alpha|^{m}}\left(\tau-\tau^{b}+\epsilon \phi_{x x}\right) .
$$

The parameter $m$ can be chosen to probe different types of behaviour. Especially, $m=0$ corresponds to the simplest possible (linear) kinetic assumption. Recall that

$$
\tau(x, t):=\frac{1}{2 b} \int_{-b}^{b} T_{12}(x, y, t) d y \quad \text { and } \quad \tau^{b}(x, t)=\frac{\partial \eta}{\partial \phi} .
$$

The non-convex energy density function is chosen to be a multiple well potential, with the plastic shear strain values at its minima representing the prefered plastic strain levels. A typical candidate could be

$$
\eta=\frac{\mu \bar{\phi}^{2}}{4 \pi^{2}}\left(1-\cos \left(2 \pi \frac{\phi}{\bar{\phi}}\right)\right) .
$$

The displacement field in the model satisfies

$$
\rho \ddot{u}_{i}=T_{i j, j} \text { in } \Omega
$$


where

$$
T_{i j}=\lambda \epsilon_{k k}^{e} \delta_{i j}+2 \mu \epsilon_{i j}^{e}
$$

$\lambda, \mu$ being the Lame parameters and

$$
\begin{aligned}
E_{i j} & :=\frac{1}{2}\left(u_{i, j}+u_{j, i}\right) \\
\epsilon_{i j}^{e} & =E_{i j} \text { in the elastic blocks, i.e. } \Omega \backslash L \\
\epsilon_{12}^{e} & =\epsilon_{21}^{e}=E_{12}-\frac{\phi}{2} ; \text { all other } \epsilon_{i j}^{e}=E_{i j} \text { in the fault layer } L,
\end{aligned}
$$

where $i, j$ take the values 1,2 . The governing equations of the system are thus:

$$
\left\{\begin{array}{l}
\rho \frac{\partial^{2} u_{i}}{\partial t^{2}}=\frac{\partial T_{i j}}{\partial x_{j}} \quad \text { in } \Omega \\
\frac{\partial \phi}{\partial t}=\frac{1}{B l^{m-1}}\left|\frac{\partial \phi}{\partial x_{1}}\right|^{2-m}\left(\tau-\tau^{b}+\epsilon \frac{\partial^{2} \phi}{\partial x_{1}^{2}}\right) \text { in } L
\end{array}\right.
$$

We make the choice $l=b$ (fault zone width in rupture dynamics; in crystals, a measure of the interatomic spacing). Then dimensional analysis suggests introducing the following dimensionless variables:

$$
\tilde{x}=\frac{x}{b}, \tilde{t}=\frac{V_{s} t}{b}, \tilde{u}=\frac{u}{b}, \tilde{T}=\frac{\boldsymbol{T}}{\mu}, \tilde{\tau^{b}}=\frac{\tau^{b}}{\mu}, \tilde{\epsilon}=\frac{\epsilon}{\mu b^{2}}, \tilde{B}=\frac{V_{s}}{\mu / B}
$$

where $\mu$ is the shear modulus and $V_{s}=\sqrt{\mu / \rho}$ is the elastic shear wave speed of the material. The non-dimensional drag number $\tilde{B}$ represents the ratio of the elastic wave speed of the material to an intrinsic velocity scale of the layer material. The non-dimensionalized version of Eqs. (3.2) reads as:

$$
\left\{\begin{array}{l}
\frac{\partial^{2} \tilde{u}_{i}}{\partial \tilde{t}^{2}}=\frac{\partial \tilde{T}_{i j}}{\partial \tilde{x}_{j}} \quad \text { in } \Omega \\
\frac{\partial \phi}{\partial \tilde{t}}=\frac{1}{\tilde{B}}\left|\frac{\partial \phi}{\partial \tilde{x}_{1}}\right|^{2-m}\left(\tilde{\tau}-\tilde{\tau}^{b}+\tilde{\epsilon} \frac{\partial^{2} \phi}{\partial{\tilde{x_{1}}}^{2}}\right) \quad \text { in } L .
\end{array}\right.
$$

We introduce a slow time scale

$$
s=\tilde{t} \Gamma, \quad \Gamma=\frac{\Gamma_{a p p l}}{\Gamma_{d y n}},
$$

where $\Gamma_{a p p l}$ is a measure of an applied overall shear strain, applied by Dirichlet boundary conditions on the displacement field $u_{1}$ on the top surface with the bottom surface held fixed, i.e. $\frac{u_{1}(x, H, t)}{2 H}=\Gamma_{a p p l}$, and $\Gamma_{d y n}$ is a constant that represents a characteristic value of $\Gamma_{a p p l}$ for which $\phi$ shows appreciable evolution in (3.3). Note that the effect of the boundary condition $\Gamma_{a p p l}$ is transmitted to the evolution of $\phi$ through $\tilde{\tau}$. Requiring $\Gamma \ll 1$ and $\Gamma \tilde{B}=1$, i.e. the applied 
averaged strain be small and the non-dimensional drag be large, we assume that $\Gamma^{2} \frac{\partial^{2} \tilde{u}_{i}}{\partial s^{2}} \ll 1$ to define a 'quasi-static' version of Eqs. (3.3):

$$
\left\{\begin{array}{l}
\frac{\partial \tilde{T}_{i j}}{\partial \tilde{x}_{j}}=0 \quad \text { in } \Omega \\
\frac{\partial \phi}{\partial s}=\left|\frac{\partial \phi}{\partial \tilde{x}_{1}}\right|^{2-m}\left(\tilde{\tau}-\tilde{\tau}^{b}+\tilde{\epsilon} \frac{\partial^{2} \phi}{\partial{\tilde{x_{1}}}^{2}}\right) \quad \text { in } L .
\end{array}\right.
$$

The system (3.3) admits initial conditions on the displacement and velocity fields $\tilde{u}_{i}, \dot{\tilde{u}}_{i}$ and the plastic strain $\phi$; the system (3.4) admits initial conditions only on $\phi$. As mentioned before, we apply the Neumann condition $\phi_{x}=0$ on the left and right boundaries of the layer $L$ and for $\left(3.3_{1}\right)$ and $\left(3.4_{1}\right)$ we utilize standard prescribed traction and/or displacement boundary conditions of linear elasticity.

We observe that for $m=2,(3.4)$ has the form of a non-local GinzburgLandau equation and for $m=1$, that of a nonlocal level set equation. For $m=0$, the case that corresponds to the simplest and most natural constitutive assumption (i.e. a linear kinetic 'law'), the equation may be considered as a generalized, nonlocal Burgers equation in Hamilton-Jacobi form.

\section{Finite Deformation FDM}

\subsection{Physical notions}

The physical model we have in mind for the evolution of the body is as follows. The body consists of a fixed set of atoms. At any given time each atom occupies a well defined region of space and the collection of these regions (at that time) is well-approximated by a connected region of space called a configuration. We assume that any two of these configurations can necessarily be connected to each other by a continuous mapping. The temporal sequence of configurations occupied by the set of atoms are further considered as parametrized by increasing time to yield a motion of the body. A fundamental assumption in what follows is that the mass and momentum of the set of atoms constituting the body are transported in space by this continuous motion. For simplicity, we think of each spatial point of the configuration corresponding to the body in the as-received state for any particular analysis as a set of 'material particles,' a particle generically denoted by $\boldsymbol{X}$.

Another fundamental assumption related to the motion of the atomic substructure is as follows. Take a spatial point $\boldsymbol{x}$ of a configuration at a given time $t$. Take a collection of atoms around that point in a spatial volume of fixed extent, the latter independent of $\boldsymbol{x}$ and with size related to the spatial scale of resolution of the model we have in mind. Denote this region as $\mathcal{D}_{c}(\boldsymbol{x}, t)$; this represents the 'box' around the base point $\boldsymbol{x}$ at time $t$. We now think of relaxing the set of atoms in $\mathcal{D}_{c}(\boldsymbol{x}, t)$ from the constraints placed on it by the rest of the atoms of the whole body, the latter possibly externally loaded. This may be 
achieved, in principle at least, by removing the rest of the atoms of the body or, in other words, by ignoring the forces exerted by them on the collection within $\mathcal{D}_{c}(\boldsymbol{x}, t)$. This (thought) procedure generates a unique placement $\mathrm{A}_{\boldsymbol{x}}$ of the atoms in $\mathcal{D}_{c}(\boldsymbol{x}, t)$ with no force in each of the atomic bonds in the collection.

We now imagine immersing $A_{x}$ in a larger collection of atoms (without superimposing any rigid body rotation), ensuring that the entire collection is in a zero-energy ground state (this may require the larger collection to be 'large enough' but not space-filling, as in the case of amorphous materials (cf. [21]). Let us assume that as $\boldsymbol{x}$ varies over the entire body, these larger collections, one for each $\boldsymbol{x}$, can be made to contain identical numbers of atoms. Within the larger collection corresponding to the point $\boldsymbol{x}$, let the region of space occupied by $\mathrm{A}_{\boldsymbol{x}}$ be approximated by a connected domain $\mathcal{D}_{r}^{\text {pre }}(\boldsymbol{x}, t)$, the latter containing the same number of atoms as in $\mathcal{D}_{c}(\boldsymbol{x}, t)$ by definition. The spatial configuration $\mathcal{D}_{r}^{\text {pre }}(\boldsymbol{x}, t)$ may correctly be thought of as stress-free. Clearly, a deformation can be defined mapping the set of points $\mathcal{D}_{c}(\boldsymbol{x}, t)$ to $\mathcal{D}_{r}^{\text {pre }}(\boldsymbol{x}, t)$. We now assume that this deformation is well approximated by a homogeneous deformation.

Next, we assume that the set of these larger collections of relaxed atoms, one collection corresponding to each $\boldsymbol{x}$ of the body, differ from each other only in orientation, if distinguishable at all. We choose one such larger collection arbitrarily, say C, keeping it fixed for all times and impose the required rigid body rotation to each of the other collections to orient them identically to C. Let the obtained configuration after the rigid rotation of $\mathcal{D}_{r}^{\text {pre }}(\boldsymbol{x}, t)$ be denoted by $\mathcal{D}_{r}(\boldsymbol{x}, t)$.

We denote the gradient of the homogeneous deformation mapping $\mathcal{D}_{c}(\boldsymbol{x}, t)$ to $\mathcal{D}_{r}(\boldsymbol{x}, t)$ by $\boldsymbol{W}(\boldsymbol{x}, t)$, the inverse elastic distortion at $\boldsymbol{x}$ at time $t$.

What we have described above is an embellished version of the standard fashion of thinking about the problem of defining elastic distortion in the classical theory of finite elastoplasticity [22], with an emphasis on making a connection between the continuum mechanical ideas and discrete atomistic ideas as well as emphasizing that no ambiguities related to spatially inhomogeneous rotations need be involved in defining the field $\boldsymbol{W}^{1}$. However, our physical construct requires no choice of a reference configuration or a 'multiplicative decomposition' of a deformation gradient defined from it into elastic and plastic parts to be invoked [10]. In fact, there is no notion of a plastic deformation $\boldsymbol{F}^{p}$ invoked in our model. Instead, as we show in Section 4.3 (4.4), an additive decomposition of the velocity gradient into elastic and plastic parts emerges naturally in this model from the kinematics of dislocation motion representing conservation of Burgers vector content in the body.

Clearly, the field $\boldsymbol{W}$ need not be a gradient of a vector field at any time.

It is important to note that if a material particle $\boldsymbol{X}$ is tracked by an individual trajectory $\boldsymbol{x}(t)$ in the motion (with $\boldsymbol{x}(0)=\boldsymbol{X}$ ), the family of neighborhoods $\mathcal{D}_{c}(\boldsymbol{x}(t), t)$ parametrized by $t$ in general can contain vastly different sets of atoms compared to the set contained initially in $\mathcal{D}_{c}(\boldsymbol{x}(0), 0)$. The intuitive idea is that

\footnotetext{
${ }^{1}$ Note that the choice of $\mathrm{C}$ affects the $\boldsymbol{W}$ field at most by a superposed spatio-temporally uniform rotation field.
} 
the connectivity, or nearest neighbor identities, of the atoms that persist in $\mathcal{D}_{c}(\boldsymbol{x}(t), t)$ over time remains fixed only in purely elastic motions of the body.

\subsection{The standard continuum balance laws}

In what follows, all spatial derivative operators are on the current configuration of the body. For any fixed set of material particles occupying the volume $B(t)$ at time $t$ with boundary $\partial B(t)$ having outward unit normal field $\boldsymbol{n}$

$$
\begin{aligned}
\overline{\int_{B(t)} \rho d v} & =0, \\
\frac{\cdot}{\int_{B(t)} \rho \boldsymbol{v} d v} & =\int_{\partial B(t)} \boldsymbol{T} \boldsymbol{n} d a+\int_{B(t)} \rho \boldsymbol{b} d v, \\
\frac{\cdot}{\int_{B(t)} \rho(\boldsymbol{x} \times \boldsymbol{v}) d v} & =\int_{\partial B(t)}(\boldsymbol{x} \times \boldsymbol{T}) \boldsymbol{n} d a+\int_{B(t)} \rho(\boldsymbol{x} \times \boldsymbol{b}) d v,
\end{aligned}
$$

represent the statements of balance of mass, linear and angular momentum, respectively. We emphasize that it is an assumption that the actual mass and momentum transport of the underlying atomic motion can be adequately represented through the material velocity and density fields governed by the above statements (with some liberty in choosing the stress tensor). For instance, in the case of modeling fracture, some of these assumptions may well require revision.

Using Reynolds' transport theorem, the corresponding local forms for these equations are:

$$
\begin{aligned}
\dot{\rho}+\rho \operatorname{div} \boldsymbol{v} & =0 \\
\rho \dot{\boldsymbol{v}} & =\operatorname{div} \boldsymbol{T}+\rho \boldsymbol{b} \\
\boldsymbol{T} & =\boldsymbol{T}^{T}
\end{aligned}
$$

The external power supplied to the body at any given time is expressed as:

$$
\begin{aligned}
P(t) & =\int_{B(t)} \rho \boldsymbol{b} \cdot \boldsymbol{v} d v+\int_{\partial B(t)}(\boldsymbol{T} \boldsymbol{n}) \cdot \boldsymbol{v} d a \\
& =\int_{B(t)} \rho \boldsymbol{v} \cdot \dot{\boldsymbol{v}} d v+\int_{B(t)} \boldsymbol{T}: \boldsymbol{D} d v
\end{aligned}
$$

where Balance of linear momentum and angular momentum have been used. On defining the kinetic energy and the free energy of the body as

$$
\begin{aligned}
K & =\int_{B(t)} \frac{1}{2} \rho \boldsymbol{v} \cdot \boldsymbol{v} d v \\
F & =\int_{B(t)} \rho \psi d v
\end{aligned}
$$


respectively, and using Reynolds' transport theorem, we obtain the mechanical dissipation

$$
\mathrm{D}:=P-\overline{K+F}=\int_{B(t)}(\boldsymbol{T}: \boldsymbol{D}-\rho \dot{\psi}) d v .
$$

The first equality above shows the distribution of applied mechanical power into kinetic, stored and dissipated parts. The second equality is used to provide guidance on constitutive structure $[10,11]$.

\subsection{Additive decomposition of the velocity gradient from the kinematics of dislocation density evolution}

The natural measure of dislocation density is

$$
\operatorname{curl} \boldsymbol{W}=-\boldsymbol{\alpha},
$$

the sign being a matter of convention related to dislocation theory. It characterizes the closure failure of integrating the inverse elastic distortion $\boldsymbol{W}$ on closed contours in the body:

$$
-\int_{a} \boldsymbol{\alpha} \boldsymbol{n} d a=\int_{c} \boldsymbol{W} d \boldsymbol{x},
$$

where $a$ is any area patch with closed boundary contour $c$ in the body. The resultant is called the Burgers vector of all dislocation lines threading the area patch; if there are no dislocation lines in the body, we are in the realm of nonlinear elasticity theory where $\boldsymbol{W}$ is the gradient of the inverse deformation on the current configuration of the body. Physically, the field $\boldsymbol{\alpha}$ is interpreted as a density of lines (threading areas) in the current configuration, carrying a vectorial attribute that reflects a jump in the 'elastic displacement field'. As such, it is reasonable to postulate, before commitment to constitutive equations, a tautological evolution statement of balance for it in the form of "rate of change = what comes in - what goes out." Following the physical reasoning in [11] for the expression of the flow of Burgers vector carried by dislocation density crossing the bounding curve of a patch $a$, we consider a conservation statement of the form

$$
\overline{\int_{a(t)} \boldsymbol{\alpha} \boldsymbol{n} d a}=-\int_{c(t)} \boldsymbol{\alpha} \times \boldsymbol{V} d \boldsymbol{x} .
$$

Here, $a(t)$ is the area-patch occupied by an arbitrarily fixed set of material particles at time $t$ and $c(t)$ is its closed bounding curve and the statement is required to hold for all such patches. $\boldsymbol{V}$ is the dislocation velocity field, physically to be understood as responsible for transporting the dislocation (line) density field in the body.

Arbitrarily fix an instant of time, say $s$, in the motion of a body and let $\boldsymbol{F}_{s}$ denote the time-dependent deformation gradient field corresponding to this motion with respect to the configuration at the time $s$. Denote positions on the configuration at time $s$ as $\boldsymbol{x}_{s}$ and the image of the area patch $a(t)$ as $a(s)$. We 
similarly associate the closed curves $c(t)$ and $c(s)$. Then, using the definition (4.1), (4.2) can be written as

$$
\begin{aligned}
\overline{\int_{a(t)} \boldsymbol{\alpha n} d a} & +\int_{c(t)} \boldsymbol{\alpha} \times \boldsymbol{V} d \boldsymbol{x}=\overline{\int_{c(t)}-\boldsymbol{W} d \boldsymbol{x}}+\int_{c(t)} \boldsymbol{\alpha} \times \boldsymbol{V} d \boldsymbol{x} \\
& =\int_{c(s)}\left[\overline{-\dot{\boldsymbol{W}} \boldsymbol{F}_{s}}+(\boldsymbol{\alpha} \times \boldsymbol{V}) \boldsymbol{F}_{s}\right] d \boldsymbol{x}_{s} \\
& =\int_{c(t)}\left[-\overline{\boldsymbol{W} \boldsymbol{F}_{s}} \boldsymbol{F}_{s}^{-1}+\boldsymbol{\alpha} \times \boldsymbol{V}\right] d \boldsymbol{x}=\mathbf{0}
\end{aligned}
$$

which implies

$$
\overline{\boldsymbol{W} \boldsymbol{F}_{s}} \boldsymbol{F}_{s}^{-1}=\boldsymbol{\alpha} \times \boldsymbol{V} \Rightarrow \dot{\boldsymbol{W}}+\boldsymbol{W} \boldsymbol{L}=\boldsymbol{\alpha} \times \boldsymbol{V},
$$

where we ignore a possibly additive gradient of a vector field, justified on the physical basis that plastic flow occurs at the microscopic scale only at points where a moving dislocation is present. This statement also corresponds to the following local statement for the evolution of $\boldsymbol{\alpha}$ :

$$
\stackrel{\circ}{\boldsymbol{\alpha}}:=(\operatorname{div} \boldsymbol{v}) \boldsymbol{\alpha}+\boldsymbol{\alpha}-\boldsymbol{\alpha} \boldsymbol{L}^{T}=-\operatorname{curl}(\boldsymbol{\alpha} \times \boldsymbol{V}) .
$$

An important feature of conservation statements for signed 'topological charge' as here is that even without explicit source terms, nucleation (of loops) is allowed. This fact, along with the coupling of $\boldsymbol{\alpha}$ to the material velocity field through the convected derivative provides an avenue for predicting homogeneous nucleation of line defects.

We note here that (4.3) can be rewritten in the form

$$
\boldsymbol{L}=\dot{\boldsymbol{F}}^{e} \boldsymbol{F}^{e-1}+\left(\boldsymbol{F}^{e} \boldsymbol{\alpha}\right) \times \boldsymbol{V}^{\alpha},
$$

where $\boldsymbol{F}^{e}:=\boldsymbol{W}^{-1}$. To make contact with classical finite deformation elastoplasticity, this may be interpreted as a fundamental additive decomposition of the velocity gradient into elastic $\left(\dot{\boldsymbol{F}}^{e} \boldsymbol{F}^{e-1}\right)$ and plastic $\left(\left(\boldsymbol{F}^{e} \boldsymbol{\alpha}\right) \times \boldsymbol{V}^{\alpha}\right)$ parts. The latter is defined by the rate of deformation produced by the flow of dislocation lines in the current configuration, without any reference to the notion of a pre-assigned reference configuration or a total plastic deformation from it (cf. [23]). We also note the natural emergence of plastic spin (i.e. a non-symmetric plastic part of $\boldsymbol{L}$ ), even in the absence of any assumptions of crystal structure but arising purely from the kinematics of dislocation motion, when a dislocation is interpreted as an elastic incompatibility. The interesting mix of exact, time-dependent, finite-deformation kinematics and kinematics specific to the phenomenon of dislocation motion leading to (4.4) is to be noted; in particular, the appearance of the velocity gradient in (4.3) almost 'out of nowhere' from considerations of Burgers vector balance.

As motivated in [11], the additive decomposition (4.4) is expected to remain valid even after averaging to macroscopic scales with the addition of an appropriate version, at finite deformations, of $\boldsymbol{L}^{p}[15]$. 


\section{References}

[1] J Lepinoux and L.P. Kubin, "The dynamic organization of dislocation structures: a simulation," Scripta metallurgica, vol. 21, no. 6, pp. 833-838, 1987.

[2] RJ Amodeo and NM Ghoniem, "Dislocation dynamics. I. A proposed methodology for deformation micromechanics," Physical Review B, vol. 41, no. 10, pp. 6958, 1990.

[3] Erik Van der Giessen and Alan Needleman, "Discrete dislocation plasticity: a simple planar model," Modelling and Simulation in Materials Science and Engineering, vol. 3, no. 5, pp. 689, 1995.

[4] Hussein M Zbib, Moono Rhee, and John P Hirth, "On plastic deformation and the dynamics of 3d dislocations," International Journal of Mechanical Sciences, vol. 40, no. 2, pp. 113-127, 1998.

[5] D Weygand, M Poignant, P Gumbsch, and O Kraft, "Three-dimensional dislocation dynamics simulation of the influence of sample size on the stress-strain behavior of fcc single-crystalline pillars," Materials Science and Engineering: A, vol. 483, pp. 188-190, 2008.

[6] Ekkehart Kröner, "Continuum theory of defects," Physics of defects, vol. 35, pp. 217-315, 1981.

[7] T Mura, "Continuous distribution of moving dislocations," Philosophical Magazine, vol. 8, no. 89, pp. 843-857, 1963.

[8] N Fox, "A continuum theory of dislocations for single crystals," IMA Journal of Applied Mathematics, vol. 2, no. 4, pp. 285-298, 1966.

[9] John R Willis, "Second-order effects of dislocations in anisotropic crystals," International Journal of Engineering Science, vol. 5, no. 2, pp. 171-190, 1967.

[10] Amit Acharya, "Constitutive analysis of finite deformation field dislocation mechanics," Journal of the Mechanics and Physics of Solids, vol. 52, no. 2, pp. 301-316, 2004.

[11] Amit Acharya, "Microcanonical entropy and mesoscale dislocation mechanics and plasticity," Journal of Elasticity, vol. 104, no. 1-2, pp. 23-44, 2011.

[12] Amit Acharya, "A model of crystal plasticity based on the theory of continuously distributed dislocations," Journal of the Mechanics and Physics of Solids, vol. 49, no. 4, pp. 761-784, 2001. 
[13] Amit Acharya, "Driving forces and boundary conditions in continuum dislocation mechanics," Proceedings of the Royal Society of London. Series A: Mathematical, Physical and Engineering Sciences, vol. 459, no. 2034, pp. 1343-1363, 2003.

[14] Amit Acharya, "New inroads in an old subject: plasticity, from around the atomic to the macroscopic scale," Journal of the Mechanics and Physics of Solids, vol. 58, no. 5, pp. 766-778, 2010.

[15] Amit Acharya and Anish Roy, "Size effects and idealized dislocation microstructure at small scales: Predictions of a phenomenological model of mesoscopic field dislocation mechanics: Part I," Journal of the Mechanics and Physics of Solids, vol. 54, no. 8, pp. 1687-1710, 2006.

[16] Marijan Babic, "Average balance equations for granular materials," International journal of engineering science, vol. 35, no. 5, pp. 523-548, 1997.

[17] R Peierls, "The size of a dislocation," Proceedings of the Physical Society, vol. 52, no. 1, pp. 34-37, 1940.

[18] Amit Acharya and Luc Tartar, "On an equation from the theory of field dislocation mechanics," Bollettino dellUnione Matematica Italiana, vol. (9) IV, pp. 409-444, 2011.

[19] Surachate Limkumnerd and James P Sethna, "Shocks and slip systems: Predictions from a mesoscale theory of continuum dislocation dynamics," Journal of the Mechanics and Physics of Solids, vol. 56, no. 4, pp. 1450$1459,2008$.

[20] James R Rice, "Inelastic constitutive relations for solids: an internalvariable theory and its application to metal plasticity," Journal of the Mechanics and Physics of Solids, vol. 19, no. 6, pp. 433-455, 1971.

[21] M. Kleman and J. . Sadoc, "A tentative description of the crystallography of amorphous solids," Journal de Physique Lettres, vol. 40, no. 21, pp. 569-574, 1979.

[22] Erastus H Lee, "Elastic-plastic deformation at finite strains," Journal of Applied Mechanics, vol. 36, no. 1, pp. 1-6, 1969.

[23] Celia Reina and Sergio Conti, "Kinematic description of crystal plasticity in the finite kinematic framework: A micromechanical understanding of $F=F^{e} F^{p}, "$ Journal of the Mechanics and Physics of Solids, vol. 67, pp. 40-61, 2014. 


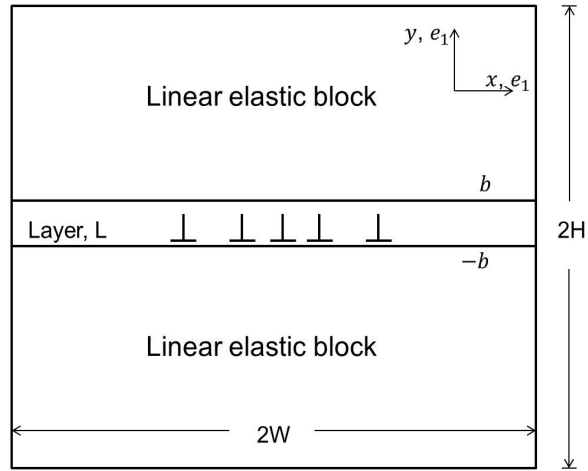

Figure 1: Geometry of the problem. 